\title{
Special issue on quantum computing with superconducting qubits
}

\author{
Alexander N. Korotkov
}

Published online: 20 February 2009

C The Author(s) 2009. This article is published with open access at Springerlink.com

Ten years ago the first superconducting qubit was demonstrated experimentally [1]. By now quantum computing with superconducting qubits has become a subject of intensive experimental and theoretical research in dozens of groups around the world. The idea of this Special Issue of the journal is to show the status of experimental research in this area after the first decade of work. Most of the best experimental groups working with superconducting qubits (with a few regrettable exceptions) are represented in this Special Issue. We hope that it gives a useful snapshot in time, demonstrating the main experimental achievements and directions of research in superconducting quantum computing.

There are many possible physical realizations of qubits [2,3]. Among the candidate systems, the obvious advantages of quantum computing with Josephson junctions are the efficient control of a quantum circuit with voltage/current/microwave pulses and use of a well-developed technology suitable for large scale integration. The fast experimental progress in experiments with superconducting qubits in the last decade confirms the importance of these advantages.

Superconducting qubits come in a variety of types, which are often separated into three categories: charge, flux, and phase qubits (though not all groups use this terminology). Single Cooper pair charge of an island carries the quantum information in the charge qubit (e.g., [1,4-16]), while the superconducting phase is the relevant degree of freedom for flux and phase qubits, which differ by the logic state encoding: two quantum levels in different wells of a potential profile are used in the flux qubit (e.g., [17-32]), and two levels in the same well are used in the phase qubit (e.g., [33-42]).

Guest Editor: Alexander N. Korotkov.

A. N. Korotkov $(\varangle)$

Department of Electrical Engineering, University of California, Riverside, CA 92521, USA

e-mail:korotkov@ee.ucr.edu 
This separation into three types is rather simplistic, and some realizations do not fit well into these categories.

By a widely accepted experimental definition, the qubit is a two-level system demonstrating Rabi oscillations. While for the first experiments with superconducting qubits even a slight evidence of Rabi oscillations was considered as a significant achievement, nowadays the "really good" Rabi oscillations imply high quality factor (long dephasing time) and a single-shot measurement with visibility approaching or exceeding $90 \%$ (e.g., [43,44]). Two-qubit logic operations have been demonstrated by a number of groups (e.g., [45-51]). There have been also experiments involving three and more superconducting qubits (e.g., $[52,53]$ ), but the logic operation of such circuits is still a challenge. Another very important problem for quantum computing is the realization of tunable coupling between qubits (e.g., [32,54-57]). A new dimension of research has been opened up with the demonstration of qubits coupled to microwave resonators (e.g., [50,51,58-64]). Besides experiments having a straightforward importance for quantum computing, many interesting experiments from a physical point of view have been realized with superconducting qubits as, for example, demonstration of Berry's phase [65], interference of Landau-Zener transitions (e.g., [13, 14, 28,29]), partial collapse [66], single-qubit lasing [61], etc. Because of the fast progress, we can hope that in a few years superconducting qubits will be sufficiently mature to be used for demonstration of simple quantum algorithms.

There are several existing reviews on superconducting quantum computing (e.g., [67-72]); however, because of the rapid progress and branching of the research subjects, it is difficult to keep up with the latest achievements. We hope that this Special Issue will be useful for readers to learn first hand about the current experimental status of the field.

I thank all the participants of this Special Issue for their great efforts. Editorial work on this special issue was supported by NSA and IARPA under ARO Grant No. W911NF-08-1-0336.

Open Access This article is distributed under the terms of the Creative Commons Attribution Noncommercial License which permits any noncommercial use, distribution, and reproduction in any medium, provided the original author(s) and source are credited.

\section{References}

1. Nakamura, Y., Pashkin, Y.A., Tsai, J.S.: Nature 398, 786 (1999)

2. Quantum Information Processing: 3, No. 1-5 (special issue) (2004)

3. Quantum Computation Roadmap: http://qist.lanl.gov/qcomp_map.shtml

4. Vion, D., Aassime, A., Cottet, A., Joyez, P., Pothier, H., Urbina, C., Esteve, D., Devoret, M.H.: Science 296, 886 (2002)

5. Collin, E., Ithier, G., Aassime, A., Joyez, P., Vion, D., Esteve, D.: Phys. Rev. Lett. 93, 157005 (2004)

6. Ithier, G., Collin, E., Joyez, P., Meeson, P.J., Vion, D., Esteve, D., Chiarello, F., Shnirman, A., Makhlin, Y., Schriefl, J., Schön, G.: Phys. Rev. B 72, 134519 (2005)

7. Duty, T., Gunnarsson, D., Bladh, K., Delsing, P.: Phys. Rev. B 69, 140503(R) (2004)

8. Wilson, C.M., Duty, T., Persson, F., Sandberg, M., Johansson, G., Delsing, P.: Phys. Rev. Lett. 98, 257003 (2007)

9. Guillaume, A., Schneiderman, J.F., Delsing, P., Bozler, H.M., Echternach, P.M.: Phys. Rev. B 69, 132504 (2004) 
10. Shaw, M.D., Schneiderman, J.F., Palmer, B., Delsing, P., Echternach, P.M.: IEEE Trans. Appl. Supercond. 17, 109 (2007)

11. Wallraff, A., Schuster, D.I., Blais, A., Frunzio, L., Majer, J., Devoret, M.H., Girvin, S.M., Schoelkopf, R.J.: Phys. Rev. Lett. 95, 060501 (2005)

12. Siddiqi, I., Vijay, R., Metcalfe, M., Boaknin, E., Frunzio, L., Schoelkopf, R.J., Devoret, M.H.: Phys. Rev. B 73, 054510 (2006)

13. Sillanpää, M., Lehtinen, T., Paila, A., Makhlin, Y., Hakonen, P.: Phys. Rev. Lett. 96, 187002 (2006)

14. Sillanpää, M., Lehtinen, T., Paila, A., Makhlin, Y., Hakonen, P.: J. Low Temp. Phys. 146, 253 (2006)

15. Schreier, J.A., Houck, A.A., Koch, Jens, Schuster, D.I., Johnson, B.R., Chow, J.M., Gambetta, J.M., Majer, J., Frunzio, L., Devoret, M.H., Girvin, S.M., Schoelkopf, R.J.: Phys. Rev. B 77, 180502(R) (2008)

16. Houck, A.A., Schreier, J.A., Johnson, B.R., Chow, J.M., Koch, J., Gambetta, J.M., Schuster, D.I., Frunzio, L., Devoret, M.H., Girvin, S.M., Schoelkopf, R.J.: Phys. Rev. Lett. 101, 080502 (2008)

17. Friedman, J.R., Patel, V., Chen, W., Tolpygo, S.K., Lukens, J.E.: Nature 406, 43 (2000)

18. Bennett, D.A., Longobardi, L., Patel V, V., Chen, W., Lukens, J.E.: Supercond. Sci. Tech. 20, S445 (2007)

19. van der Wal, C.H., ter Haar, A.C.J., Wilhelm, F.K., Schouten, R.N., Harmans, C.J.P.M., Orlando, T.P., Lloyd, S., Mooij, J.E.: Science 290, 773 (2000)

20. Chiorescu, I., Nakamura, Y., Harmans, C.J.P.M., Mooij, J.E.: Science 299, 1869 (2003)

21. Chiorescu, I., Bertet, P., Semba, K., Nakamura, Y., Harmans, C.J.P.M., Mooij, J.E.: Nature 431, 159 (2004)

22. Lupaşcu, A., Saito, S., Picot, I., De Groot, P.C., Harmans, C.J.P.M., Mooij, J.E.: Nat. Phys. 3, 119 (2007)

23. Yoshihara, F., Harrabi, K., Niskanen, A.O., Nakamura, Y., Tsai, J.S.: Phys. Rev. Lett. 97, 167001 (2006)

24. Saito, S., Meno, T., Ueda, M., Tanaka, H., Semba, K., Takayanagi, H.: Phys. Rev. Lett. 96, 107001 (2006)

25. Kakuyanagi, K., Meno, T., Saito, S., Nakano, H., Semba, K., Takayanagi, H., Deppe, F., Shnirman, A.: Phys. Rev. Lett. 98, 047004 (2007)

26. Il'ichev, E., Oukhanski, N., Izmalkov, A., Wagner, Th., Grajcar, M., Meyer, H.-G., Smirnov, A.Y., van den Brink, A.M., Amin, M.H.S., Zagoskin, A.M.: Phys. Rev. Lett. 91, 097906 (2003)

27. Izmalkov, A., van der Ploeg, S.H.W., Shevchenko, S.N., Grajcar, M., Il'ichev, E., Huebner, U., Omelyanchouk, A.N., Meyer, H.G.: Phys. Rev. Lett. 101, 017003 (2008)

28. Oliver, W.D., Yu, Y., Lee, J.C., Berggren, K.K., Levitov, L.S., Orlando, T.P.: Science 310, 1653 (2005)

29. Berns, D.M., Rudner, M.S., Valenzuela, S.O., Berggren, K.K., Oliver, W.D., Levitov, L.S., Orlando, T.P.: Nature 455, 7209 (2008)

30. Valenzuela, S.O., Oliver, W.D., Berns, D.M., Berggren, K.K., Levitov, L.S., Orlando, T.P.: Science 314, 1589 (2006)

31. Plourde, B.L.T., Robertson, T.L., Reichardt, P.A., Hime, T., Linzen, S., Wu, C.-E., Clarke, J.: Phys. Rev. B 72, 060506 (2005)

32. Harris, R., Berkley, A.J., Johnson, M.W., Bunyk, P., Govorkov, S., Thom, M.C., Uchaikin, S., Wilson, A.B., Chung, J., Holtham, E., Biamonte, J.D., Smirnov, A.Y., Amin, M.H.S., van den Brink, A.M.: Phys. Rev. Lett. 98, 177001 (2007)

33. Yu, Y., Han, S.Y., Chu, X., Chu, S.I., Wang, Z.: Science 296, 889 (2002)

34. Martinis, J.M., Nam, S., Aumentado, J., Urbina, C.: Phys. Rev. Lett. 89, 117901 (2002)

35. Cooper, K.B., Steffen, M., McDermott, R., Simmonds, R.W., Oh, S., Hite, D.A., Pappas, D.P., Martinis, J.M.: Phys. Rev. Lett. 93, 180401 (2004)

36. Berkley, A.J., Xu, H., Ramos, R.C., Gubrud, M.A., Strauch, F.W., Johnson, P.R., Anderson, J.R., Dragt, A.J., Lobb, C.J., Wellstood, F.C.: Science 300, 1548 (2003)

37. Xu, H., Strauch, F.W., Dutta, S.K., Johnson, P.R., Ramos., R.C., Berkley, A.J., Paik, H., Anderson, J.R., Dragt, A.J., Lobb, C.J., Wellstood, F.C.: Phys. Rev. Lett. 94, 027003 (2005)

38. Dutta, S.K., Strauch, F.W., Lewis, R.M., Mitra, K., Paik, H., Palomaki, T.A., Tiesinga, E., Anderson, J.R., Dragt, A.J., Lobb, C.J., Wellstood, F.C.: Phys.Rev. B 78, 104510 (2008)

39. Claudon, J., Balestro, F., Hekking, F.W.J., Buisson, O.: Phys. Rev. Lett. 93, 187003 (2004)

40. Claudon, J., Fay, A., Hoskinson, E., Buisson, O.: Phys. Rev. B 76, 024508 (2007)

41. Oh, S., Cicak, K., Kline, J.S., Sillanpää, M.A., Osborn, K.D., Whittaker, J.D., Simmonds, R.W., Pappas, D.P.: Phys. Rev. B 74, 100502 (2006)

42. Lisenfeld, J., Lukashenko, A., Ansmann, M., Martinis, J.M., Ustinov, A.V.: Phys. Rev. Lett. 99, 170504 (2007) 
43. Lupaşcu, A., Driessen, E.F.C., Roschier, L., Harmans, C.J.P.M., Mooij, J.E.: Phys. Rev. Lett. 96, 127003 (2006)

44. Martinis J.M.: in this issue. doi:10.1007/s11128-009-0104-2

45. Pashkin, Y.A., Yamamoto, T., Astafiev, O., Nakamura, Y., Averin, D.V., Tsai, J.S.: Nature 421, 823 (2003)

46. Yamamoto, T., Pashkin, Y.A., Astafiev, O., Nakamura, Y., Tsai, J.S.: Nature 425, 941 (2003)

47. McDermott, R., Simmonds, R.W., Steffen, M., Cooper, K.B., Cicak, K., Osborn, K.D., Oh, S., Pappas, D.P., Martinis, J.M.: Science 307, 5713 (2005)

48. Steffen, M., Ansmann, M., Bialczak, R.C., Katz, N., Lucero, E., McDermott, R., Neeley, M., Weig, E.M., Cleland, A.N., Martinis, J.M.: Science 313, 1423 (2006)

49. Plantenberg, J.H., de Groot, P.C., Harmans, C.J.P.M., Mooij, J.E.: Nature 447, 836 (2007)

50. Sillanpää, M.A., Park, J.I., Simmonds, R.W.: Nature 449, 438 (2007)

51. Majer, J., Chow, J.M., Gambetta, J.M., Koch, Jens, Johnson, B.R., Schreier, J.A., Frunzio, L., Schuster, D.I., Houck, A.A., Wallraff, A., Blais, A., Devoret, M.H., Girvin, S.M., Schoelkopf, R.J.: Nature 449, 443 (2007)

52. Grajcar, M., Izmalkov, A., van der Ploeg, S.H.W., Linzen, S., Plecenik, T., Wagner, T., Hubner, U., Il'ichev, E., Meyer, H.G., Smirnov, A.Y., Love, P.J., van den Brink, A.M., Amin, M.H.S., Uchaikin, S., Zagoskin, A.M.: Phys. Rev. Lett. 96, 047006 (2006)

53. Niskanen, A.O., Harrabi, K., Yoshihara, F., Nakamura, Y., Tsai, J.S.: Phys. Rev. B 74, 220503(R) (2006)

54. Hime, T., Reichardt, P.A., Plourde, B.L.T., Robertson, T.L., Wu, C.-E., Ustinov, A.V., Clarke, J.: Science 314, 1427 (2006)

55. Niskanen, A.O., Harrabi, K., Yoshihara, F., Nakamura, Y., Lloyd, S., Tsai, J.S.: Science 316, 723726 (2007)

56. van der Ploeg, S.H.W., Izmalkov, A., van den Brink, A.M., Hübner, U., Grajcar, M., Il'ichev, E., Meyer, H.-G., Zagoskin, A.M.: Phys. Rev. Lett. 98, 057004 (2007)

57. Fay, A., Hoskinson, E., Lecocq, F., Levy, L.P., Hekking, F.W.J., Guichard, W., Buisson, O.: Phys. Rev. Lett. 100, 187003 (2008)

58. Wallraff, A., Schuster, D.I., Blais, A., Frunzio, L., Huang, R.S., Majer, J., Kumar, S., Girvin, S.M., Schoelkopf, R.J.: Nature 431, 162 (2004)

59. Houck, A.A., Schuster, D.I., Gambetta, J.M., Schreier, J.A., Johnson, B.R., Chow, J.M., Frunzio, L., Majer, J., Devoret, M.H., Girvin, S.M., Schoelkopf, R.J.: Nature 449, 328 (2007)

60. Johansson, J., Saito, S., Meno, T., Nakano, H., Ueda, M., Semba, K., Takayanagi, H.: Phys. Rev. Lett. 96, 127006 (2006)

61. Astafiev, O., Inomata, K., Niskanen, A.O., Yamamoto, T., Pashkin , Y.A., Nakamura, Y., Tsai, J.S.: Nature 449, 588 (2007)

62. Hofheinz, M., Weig, E.M., Ansmann, M., Bialczak, R.C., Lucero, E., Neeley, M., O'Connell, A.D., Wang, H., Martinis, J.M., Cleland, A.N.: Nature 454, 310 (2008)

63. Fink, J.M., Göppl, M., Baur, M., Bianchetti, R., Leek, P.J., Blais, A., Wallraff, A.: Nature 454, 315 (2008)

64. Palacios-Laloy A. et al.: to be published

65. Leek, P.J., Fink, J.M., Blais, A., Bianchetti, R., Goppl, M., Gambetta, J.M., Schuster, D.I., Frunzio, L., Schoelkopf, R.J., Wallraff, A.: Science 318, 1889 (2007)

66. Katz, N., Ansmann, M., Bialczak, R.C., Lucero, E., McDermott, R., Neeley, M., Steffen, M., Weig, E.M., Cleland, A.N., Martinis, J.M., Korotkov, A.N.: Science 312, 1498 (2006)

67. Makhlin, Y., Schön, G., Shnirman, A.: Rev. Mod. Phys. 73, 357 (2001)

68. Averin, D.V.: Fortschr. Phys. 48, 1055 (2000)

69. Devoret, M.H., Martinis, J.M.: Quant. Inform. Process. 3, 163 (2004)

70. You, J.Q., Nori, F.: Phys. Today 58(11), 42 (2005)

71. Schoelkopf, R.J., Girvin, S.M.: Nature 451, 664 (2008)

72. Clarke, J., Wilhelm, F.K.: . Nature 453, 1031 (2008) 